\title{
L'“angiola giovanissima” e il linguaggio degli angeli: la musica della e nella Vita Nova
}

\author{
Barbara Kuhn \\ Universität Eichstaett \\ Barbara.Kuhn@ku.de
}

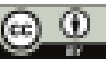

\section{Riassunto}

Partendo dal fatto paradossale che la Vita Nova possa essere definito un "Buch der Lieder" (Wehle) o "libro dei canti", pur parlando della musica in senso stretto solo in due luoghi, il presente contributo intende esaminare il rapporto esistente fra il libro e la "musica" in un senso più ampio e proporre una lettura della Vita Nova che mette l'accento proprio sulla musicalità delle poesie dantesche e le sue implicazioni. Secondo la convinzione medievale il linguaggio degli angeli non è altro che musica, mentre da parte sua la musica riesce a comunicare già sulla terra un'idea del canto degli angeli. Nel testo dantesco, non solo la canzone è 'angelo' nel senso etimologico del termine, essendo mandata dall'io a portare un messaggio ai destinatari; non solo porta metaforicamente l'io, la cui lingua parla "quasi come per se stessa mossa”, al cielo. Innanzitutto, è la poesia che con la sua suavitas riesce a superare i limiti del linguaggio umano e a procurare la beatitudine, trasportando coloro che la sanno udire già in vita "oltre la spera".

Parole chiave: Vita Nova, musica, angelo, linguaggio degli angeli, musica delle parole.

\begin{abstract}
Paradoxically, the Vita Nova mentions music in its strict signification only twice, and nonetheless it can be defined as a "Buch der Lieder" (Wehle) or "book of songs". So this paper examines the relationship between the book and the "music" in a broader sense of the word and proposes an interpretation of the Vita Nova that focuses on the musicality of the poems and its implications. According to the medieval conviction, the language of the angels is nothing but music, and therefore on earth it is only music that can give us an idea of the singing of the angels. In Dante's text not only the canzone is an 'angel' in the etymological sense of the word, because it carries a message to the addressees; not only does it transport the I in a metaphorical sense to heaven because his tongue speaks "quasi come per se stessa mossa". Above all poetry with its suavitas can transcend the limits of human language and give the beatitudo or transport "oltre la spera" those who are able to understand it already during their lifetime.
\end{abstract}

Keywords: Vita Nova, music, angel, language of the angels, music of words. 
Dante il mover gli diè del cherubino e d'aere azzurro e d'òr lo circonfuse Giosuè Carducci, Il sonetto

A prima vista potrebbe sembrare strano voler concentrare l'attenzione sulla Vita Nova, se ci si propone di riflettere sul tema Dante e la musica - almeno se si dà uno sguardo alla critica in quest'ambito. Dei diversi contributi dedicati alla concezione medievale della musica e a La musica nel tempo di Dante $-{ }^{2}$ questo il titolo di un convegno i cui atti sono stati pubblicati nel I988 -, la maggior parte o studia come la musica viene tematizzata in senso proprio o anche figurato nella Commedia, ${ }^{3}$ o esamina le riflessioni sulla musica presenti nel De vulgari eloquentia e nel Convivio, ${ }^{4}$ riguardanti innanzitutto il rapporto tra poesia e musica, anche qui sia in senso proprio che figurato. Eccetto alcuni riferimenti puntuali a certi passi con lo scopo di avvalorare le relative argomentazioni dei diversi studi, stupisce il fatto che proprio la Vita Nova, il "Buch der Lieder" o "libro dei canti" secondo Winfried Wehle, non venga contemplato da chi si occupa di questo tema. Persino un saggio intitolato "Presenze musicali nelle opere minori di Dante" (De Benedictis 2000: 35-56) limita il suo orizzonte di ricerca al Convivio, al De vulgari eloquentia e al De Monarchia. Invece già i diversi generi poetici, che compongono la Vita Nova, suggeriscono una relazione con la musica. In effetti l'io narrante raccoglie nel suo "libello" una ballata, delle canzoni e dei sonetti, testi cioè che si definiscono in un modo o nell'altro grazie al loro legame con la musica (cfr. anche King 1974: 5I). Già ad un primo sguardo ci si rende conto che questi stessi testi non sono semplicemente delle riflessioni sulla poesia d'amore in forma lirica, ma anche e soprattutto dei veri e propri canti, più precisamente delle lamentazioni e degli inni di lode, come del resto è indicato sia dal programmatico "stilo de la loda", sia dagli incipit quali Piangete, amanti o L'amaro lagrimar. Tanto i generi quanto gli oggetti delle poesie invitano dunque ad approfondire la questione del ruolo svolto dalla musica della e nella Vita Nova, creando, un'altra volta, un moto di stupore in chi legge.

I. L'articolo che segue costituisce una versione tradotta, rivista e aggiornata di Kuhn 2009; ringrazio cordialmente Beatrice Baldarelli per aver corretto e limato la mia traduzione del testo originale.

2. Cfr. ad es. Hammerstein 21990; Pestalozzi 1988; Russo 1988; Richter 1988; Kropfinger 1988; Bruggisser-Lanker 2010.

3. Cfr. ad es. Pazzaglia 1986; Iannucci 1989; Morelli 1989, nonché Armour 1997; Barański 1997; Basile 1997; Dovara in Varoli Piazza 1997; Jones 2010; Verdicchio 20I0; Schneider 2012. Cfr. inoltre Pistelli Rinaldi 1968, che dopo due brevi capitoli intitolati "La musica del tempo di Dante" e "La musicalità di Dante nelle opere minori" (soprattutto De vulgari eloquentia e Convivio), si dedica a "La musicalità della Commedia". Anche la questione degli angeli nell'universo dantesco viene trattata innanzitutto in rapporto alla Commedia: cfr. Boyde 1984: 287-329; Petrocchi 1994.

4. Cfr. ad es. Monterosso 1965; Pazzaglia 1988; Pazzaglia 1967.

5. "Ein Buch der Lieder" è il titolo del secondo capitolo di Wehle i986: 15-30. 
Mentre, ad esempio nella Commedia, soprattutto nel Purgatorio e nel Paradiso, si canta in diversi luoghi, ${ }^{6}$ la Vita Nova, benché si presenti dall'inizio alla fine formalmente come un "libro dei canti", fa raramente cenno al cantare o alla musica nell'accezione propria o moderna della parola. Segnatamente solo due luoghi la evocano esplicitamente. Il primo è la già menzionata ballata che contiene la presentazione musicale della lirica e che costituisce una specie di hapax nel prosimetrum, non solo per la specificità di questo genere tanto amato dagli stilnovisti, che qui appare un'unica volta, ma soprattutto per l'allusione alla performance delle poesie. ${ }^{7}$ Con questa ballata l'amante, per ordine di Amore, chiede perdono all'amata per il fraintendimento derivato dal suo comportamento; tutte le sue poesie erano indirizzate alle varie donne-schermo e non alla donna veramente amata, per fingersi agli occhi di tutti come amante incostante. Poiché la stessa Beatrice gli rifiuta ora il suo saluto beatifico, Amore gli consiglia di porre fine agli infingimenti, di lasciar perdere i simulacra e di cantare veramente la donna amata. È solo a questa svolta della storia d'amore e della storia del poetare che Amore raccomanda di far accompagnare le parole dalla musica - "falle adornare di soave armonia" (5.15/ xii 8),$-{ }^{8}$ ed è solo in questa poesia che l'io, parlando del suo componimento, ne menziona il "dolze sono" (v. I5) e la "nota soave" (v. 38 ).

Il secondo episodio della Vita Nova che evoca il cantare fa parte di una delle visioni dell'io narrante, e anche questa occorrenza si trova in un luogo essenziale per l'intero testo. Si tratta del presentimento della morte di Beatrice, manifestatosi nel corso della malattia dell' io, il quale sogna prima la propria morte e poi quella dell'amata:

imaginai alcuno amico che mi venisse a dire: "Or non sai? la tua mirabile donna è partita di questo secolo". Allora cominciai a piangere molto pietosamente; e non solamente piangea ne la imaginazione, ma piangea con li occhi, bagnandoli di vere lagrime. Io imaginava di guardare verso lo cielo, e pareami vedere moltitudine d'angeli li quali tornassero in suso, ed aveano dinanzi da loro una nebuletta bianchissima. A me parea che questi angeli cantassero gloriosamente, e le parole del loro canto mi parea udire che fossero queste: Osanna in excelsis; e altro non mi parea udire (I4.5-7 / xxiii 6-7).

Quantunque si tratti di un'“erronea fantasia” o, per dirla con le parole della poesia seguente, di un "imaginar fallace”, tuttavia l'inno udito in sogno, ese-

6. L'Inferno invece è caratterizzato da altri suoni: cfr. tra l'altro Sanguineti 1988, nonché Schneider 2OI2: II3-II5.

7. Per quanto riguarda la particolarità della ballata cfr. anche Carrai 2006: 94-96.

8. Visto che a causa del problema irrisolto di un'edizione critica definitiva, citare la Vita Nova è una questione spinosa, menziono ogni volta il capitolo (come Gorni 2006), prima secondo l'edizione curata da Gorni e poi secondo quella curata da Barbi, perché continua a costituire la base di quasi tutte le edizioni moderne, nonché di gran parte della critica dantesca. Come Gorni 2006 stesso, scelgo anch'io la grafia proposta da Barbi e ripresa dall'edizione curata da Colombo, e non quella latineggiante dell'edizione di Gorni. 
guito da un coro di angeli al momento della morte di Beatrice, dà un segnale importante sia riguardo al ruolo giocato dalla musica in questo testo, sia al legame esistente tra Beatrice e il "linguaggio degli angeli", espresso nell' Osanna.

Da una parte, il fatto che la ballata sia accompagnata da musica e contenga il canto degli angeli al momento dell'ascensione di Beatrice, è particolarmente significativo non solo perché sono questi due gli unici passi della Vita Nova ad evocare la musica in modo esplicito, ma soprattutto perché spiccano rispetto al resto, conferendo un rilievo particolare agli episodi che stanno alla base dello snodo e del sogno premonitore. Dall'altra parte, però, non è detto che solo qui e solo in questo senso la musica vi svolga un ruolo. Al contrario, proprio l'apparente paradosso che un 'libro dei canti' parli così poco del cantare e della musica, ci suggerisce che il concetto di musica, secondo l'accezione odierna, non esaurisca tutti i significati che la parola poteva avere nel Medioevo. È probabile che i due passi alludano a due altri aspetti più ampi che devono essere considerati. Da un lato, il canto degli angeli nella canzone di mezzo fa pensare proprio a quel "linguaggio degli angeli" di cui si parlerà nella prima parte del De vulgari eloquentia, che rimanda alla presenza e all'azione degli angeli nella Vita Nova, in cui fin dall'inizio Beatrice stessa è presentata come "angiola giovanissima". Dall'altro, per quanto ancora legato alla questione degli angeli, il riferimento alla "soave armonia" e al "dolze sono" colloca l'idea di suono e di armonia al di fuori del loro significato letterale, in un ambito più vasto della concezione medievale della musica, ma anche in quello più specifico di 'musica verbale'. Di quest'ultima si occupa anche la seconda parte del De vulgari eloquentia. Vedremo comunque che essa è molto legata anche alla Vita Nova. ${ }^{9}$

9. La maggior parte dei critici che trattano il vasto campo di 'Dante e la musica' si concentrano sulla "tematica musicale" (Cappuccio 2008: I49) nei testi danteschi, anche quando si parla in modo più generale dell'"elemento musicale" o di "immagini musicali" - così Cappuccio (2005; 2008; 2009) cerca ad esempio di distinguere il tipo di musica, polifonia o monodia, di cui si tratta nelle allusioni della Commedia, mentre Schneider (20IO; 20I2) si interessa soprattutto alla funzione drammatica della musica nelle diverse cantiche). La musicalità del linguaggio non costituisce che raramente il centro dell'interesse, e ciò malgrado le posizioni formulate da Dante in De vulgari eloquentia e citate da numerosi critici (cfr. invece King 1974: 53, che evoca la musicalità della poesia dovuta alla scelta delle parole e ricorda "the crucial importance of word sound for the achievement of poetic meaning"). Molto interessante invece è l'articolo di Williamson su "Sensory Experience in Medieval Devotion: Sound and Vision, Invisibility and Silence", che tratta delle "relationships between art and music", ad esempio della percezione visiva della musica in alcuni quadri, la quale spesso include una specie di percezione auditiva nell'immaginazione. Williamson menziona tra l'altro, in analogia con 'l'occhio interiore', "the 'inner eyes' (or 'eyes of the mind,' 'eyes of the heart,' or 'eyes of the soul')", anche 'l'orecchio del cuore', "the ear of the heart" (Williamson 20I3: I3), importantissimo anche per la lettura dei testi danteschi e per la percezione della loro musicalità inerente. Vale anche per l'opera dantesca quello che Williamson afferma sulla devozione medievale in genere: bisogna considerare l'uomo non in modo dualistico, diviso in corpo e spirito, ma come un insieme, proprio come in Dante "bontade" e "bellezza" delle poesie diventano inseparabili. 


\section{Gli ANGEli della Vita Nova}

Che Beatrice sia un angelo o meno, è una delle questioni su cui la critica dantesca ha ripetutamente insistito dalle origini fino ad oggi. Così ad esempio Guglielmo Gorni, nel commento all'edizione della Vita Nova da lui curata nel 1996, sostiene la tesi che, descrivendo delle figure angeliche dopo la morte di Beatrice, l'io del testo aspirerebbe certo a "un recupero almeno delle angeliche sembianze della defunta"; tuttavia "Beatrice, che non è mai assimilata a un angelo in vita, se non per metafora [..., è donna, per l'appunto, e non angelo: donna gloriosa, beata, benedecta, non puro spirito" (cfr. Gorni 1996: 269 sgg.). Marco Santagata invece, in Amate e amanti pubblicato nel 1999, arriva a una conclusione diametralmente opposta, benché anch'esso parta dalla considerazione che i luoghi nei quali Beatrice viene chiamata angelo siano assai rari in significativo contrasto con le tante "donne angelo" della poesia in volgare di tutte le epoche. Proprio perché la "donna angelo", come dice Pasquini, pare un motivo quasi endemico nella poesia stilnovistica e una delle "idee-forza" di quell'avanguardia poetica che trasforma in analogia la metafora conosciutissima della donna angelicata cantata da un Giacomo da Lentini o da un Guittone d'Arezzo (cfr. Pasquini 1995: 7II-7I3), Dante invece, sempre secondo le riflessioni di Santagata, vuole espressamente mettere in risalto l'unicità della 'sua' donna, opponendola alla schiera di donne fatte oggetto di canti, alle "innumerevoli repliche e variazioni" del topos, come esso si presenta fin da Guinizelli. ${ }^{10}$ Così alla rara occorrenza di 'angelo', termine con cui tradizionalmente ci si rifaceva alla soprannaturalità della donna", si opporrebbe quella sorprendentemente frequente di Beatrice come "miracolo", concetto "che è invece quasi sconosciuto alla lirica profana". Secondo il critico, è innanzitutto il linguaggio dei numeri che conferisce a Beatrice una "divinità matematica" accanto a quella metaforica della poesia, cioè accanto a "uno dei più confusi e consolidati luoghi comuni della lirica romanza", perché "con [questa] lingua, diversa da quella della poesia, Dante ribadisce quel tema dell'origine divina della dama" e ne fa una cosa nuova, inusitata: "Mediante questo linguaggio, infatti, Dante può comunicare ai lettori che Beatrice non è come un angelo, ma è un angelo; non è un oggetto di meraviglia come fosse un miracolo, ma è un miracolo" (cfr. Santagata I999: 13-24).

Più importante però dello stabilire a priori se Beatrice "è donna, per l'appunto, e non angelo" oppure se "non è come un angelo, ma è un angelo", più

IO. Guinizelli con la sua descrizione della donna in Al cor gentil (v. 58: "[t]enne d'angel sembianza”) diventa modello ad esempio per l'“angelica sembianza" (v. 19) in Fresca rosa novella di Cavalcanti, per l'Angelica figura novamente nonché "quest'angela che par di ciel venuta" (v. 7) in Dolcè 'l pensier che mmi notrica 'l core di Lapo Gianni; per "Angel di Dio simiglia in ciascun atto / questa giovane bella" (vv. I-2) di Cino da Pistoia, e per molti altri testi stilnovistici. 
importante di un ragionamento definitivamente pro o contra, sembra il fatto che nel testo siano riconosciuti alla donna gli attributi e l'agire di un essere soprannaturale o di un angelo. ${ }^{\text {II }}$ Così fin dal primo incontro non solo è per l'io "angiola giovanissima", ma è da Amore presentata come la "beatitudo vestra". Conseguentemente nove anni più tardi il saluto della "mirabile donna" procurerà all'io la beatitudine che rimarrà uno dei leitmotiv del testo fino alla scoperta dello "stilo de la sua lode" (17.4 / xxvi 4):

passando per una via, volse li occhi verso quella parte ov' io era molto pauroso, e per la sua ineffabile cortesia, la quale è oggi meritata nel grande secolo, mi salutoe molto virtuosamente, tanto che me parve allora vedere tutti li termini de la beatitudine. L'ora che lo suo dolcissimo salutare mi giunse, era fermamente nona di quello giorno; e però che quella fu la prima volta che le sue parole si mossero per venire a li miei orecchi, presi tanta dolcezza, che come inebriato mi partio da le genti, e ricorsi a lo solingo luogo d'una mia camera, e puosimi a pensare di questa cortesissima. E pensando di lei, mi sopragiunse uno soave sonno, ne lo quale m'apparve una maravigliosa visione [...] (I.I2-I4 / iii I-3).

Si tratta della seconda apparizione della "gloriosa donna de la mia mente". Il primo incontro in età infantile aveva già provocato il suo rapimento totale, l'abbandono di tutti gli spiriti della vita e la presa di potere da parte di Amore. Tuttavia quella prima descrizione, secondo le parole del narratore, poteva passare ancora per un "parlare fabuloso". Qui invece dove per la prima volta Beatrice si rivolge all'amante e all'impressione visiva s'aggiunge quella auditiva, il testo raccoglie una molteplicità di elementi che connotano il carattere angelico della donna: dal suo passargli davanti (il "passage angélique") fino al "dolcissimo salutare", la cui soavità lo rende "come inebriato". Questa "parole angélique", che riesce a trasportare in altri mondi colui che la ode, ${ }^{\mathrm{I} 2}$ genera la prima di una serie di visioni che costelleranno il testo fino alla sua conclusione: il sogno premonitore nel quale Beatrice, mangiando il cuore dell'amante, è portata al cielo da Amore su una nuvola di fuoco.

II. Cfr. Ginsberg (1999: 39), che da un lato sottolinea la differenza rispetto a Guinizelli, dall'altro lato, basandosi di nuovo sul desiderio degli angeli in cielo, espresso in Donne ch'avete, di avere Beatrice con loro, menziona la vicinanza tra la Beatrice 'luminosa' e gli angeli: "The angel's words, however, do not arise, as ours do, from seeing Beatrice's body or its image but as a response to her marvelous being that is light [cfr. vv. I6-18...]. Light enables understanding; in a play of speculation that makes her one with heaven's intelligences, Beatrice's light is reflected in the light that is the angel's utterance in the divine intellect" (Ginsberg 1999: 59).

I2. "La parole angélique restaure une événementialité et un possible. En ce sens, elle est 'métaphorique': passage à un autre genre, invention d'un autre espace, création d'un possible à l'intérieur de ce que les faits posent comme impossible. Dans un songe, Jacob 'voit' des anges montant et descendant les degrés de l'échelle qui mesure des distances et des médiations nécessaires entre la terre et le ciel. L'espace du songe donne ainsi à la 'métaphore' angélique sa figure fondamentale: une parole 'passe' les frontières des êtres ou des choses, restaure paradoxalement une historicité dans le cadre des lois cosmiques et, sous le signe du subit et de l'insolite, ouvre un champ de possible" (Certeau I984: 9). 
Non è solo il terrore dell'io, il suo "heilige[s] Erschrecken vor der Schönheit", a trasformare la struttura della sua coscienza, la "Bewußtseinsstruktur", 13 non è solo il saluto a condurlo ai confini estremi della "beatitudine"; è anche l'“ineffabile cortesia" a evocare un fatto soprannaturale. L'ineffabilità e la "dulcedo", concetti correnti nella mistica latina, qui appaiono insieme probabilmente per la prima volta in un testo in volgare romanzo: ${ }^{14}$ In questo modo sono come il segnale che non si tratta semplicemente di un topos ripreso passivamente dalla tradizione mistica. Averlo inserito all'interno della storia di una 'vita nuova' comporta una sua specifica configurazione e valorizzazione, proprio come nel caso del carattere angelico della donna in quanto ripresa del topos della 'donna angelicata', topos ricorrente ad esempio nella poesia in volgare e trasformato nel e dal testo dantesco.

Servendosi di svariati elementi tematici e di procedimenti diversi, il testo sottolinea ripetutamente il carattere angelico, l'apparizione e l'azione soprannaturali di Beatrice. Si pensi soltanto al rilievo che assume fin dall'inizio il "nove", numero che, nell'ottica del simbolismo numerico, conferisce un carattere soprannaturale al personaggio. Si pensi al noto parallelismo tra Beatrice e Cristo, alla sua azione miracolosa, al fatto che venga non solo cantata, ma addirittura reclamata in cielo dagli angeli prima ancora di morire. ${ }^{\mathrm{IS}} \mathrm{Si}$ pensi infine all'explicit del testo, secondo cui la "benedetta Beatrice [...] gloriosamente

13. 'Die Liebe und die 'andere Welt' kommen zu uns nicht nach unserem Willen. [...] Wenn man daran denkt, wie dieses Geschehen von außen als eine Änderung der menschlichen Bewußtseinsstruktur erscheint, so wird man vergleichend sagen können, daß solche Änderungen ebensowenig unserem Willen unterworfen seien, wie etwa das Wachstum oder die heilenden Kräfte unseres Körpers. [...] Plato sagt, daß die Liebe die Sehnsucht der Menschen nach der Unsterblichkeit sei, und daß jenes heilige Erschrecken vor der Schönheit zugleich ein Erschrecken vor der Unendlichkeit sei, die uns dabei plötzlich vor das Bewußtsein tritt. Vielleicht darf man das auch so aussprechen, daß nicht nur in der Liebe, sondern in all den Augenblicken, in denen uns die 'andere Welt' begegnet, in unserem Bewußtsein ein Gefühl für jenen unendlichen Lebensprozeß erwacht, an dem wir für eine kurze Zeitspanne teilnehmen und der sich an uns und über unser irdisches Dasein hinweg vollzieht" (Heisenberg 1989: I63 e I66). Per quanto riguarda l'equazione bellezzaterrore e soprattutto il terrore provocato da Beatrice, cfr. inoltre: "Erzittern der Sinne und der Seele, Erbleichen, Schrecken, Blendung des Auges, Ohnmacht der Sprache, und, durch dies alles hindurch, Veredelung dessen, der sie erblickt: das ist ihre Wirkung, die durch viele Grade der Heftigkeit wie der Beseligung reicht. Schönheit als Schrecknis [...] birgt die große Paradoxie, daß der Erschreckte, ja Zerstörte nicht den Wunsch nach Beseitigung der zerstörenden Ursache, vielmehr den anderen nach ihrer Erhaltung hegt. Der Wortschatz für das Erschrecken, das von Beatrice ausgeht, ist größer als derjenige für die Beglückung. Aber diese Tatsache steht absichtsvoll im umgekehrten Verhältnis zum Reichtum der Beglückung: denn für diese will die Sprache ärmer sein, um mittels der Unsagbarkeit eben ihren Reichtum auszudrücken" (Friedrich 1964: II4).

I4. Cfr. Colombo 1987: 73-89 et passim, nonché le note dettagliate al testo della Vita Nuova nell'edizione da lei curata (Colombo 2008: 40 e I36-138).

15. Anche in questo dettaglio si cela un'analogia con Cristo, che gli angeli desiderano vedere, secondo l'angelologia di San Bernardo di Chiaravalle (cfr. Knoch 2006: I4), proprio come nella canzone Donne ch'avete (vv. I5-2I) gli angeli preferirebbero godere della compagnia di quell'essere di luce che è Beatrice invece di saperla sempre sulla terra. 
mira ne la faccia di colui qui est per omnia secula benedictus" (3I.3 / xlii 3), frase questa che per mezzo del 'polittoto bilingue' di benedetta e benedictus riesce a superare il confine tra volgare e latino, tra mondo profano e mondo sacro, creando così un legame univoco tra Beatrice e Cristo per rimarcare una volta di più l'essenza angelica della donna, in un'evocazione degli angeli miranti in eterno il volto del Padre in cielo, che sembra tratta quasi alla lettera dal vangelo di Matteo (I8, Io)..$^{16}$

Non solo l'io narrante ma tutti coloro davanti ai quali passa Beatrice riconoscono la sua bellezza sovrumana, che occupa le vette persino delle gerarchie angeliche: "Questa non è femmina, anzi è uno de li bellissimi angeli del cielo." Deve essere dunque annoverata, secondo l'interpretazione di Gorni, ${ }^{17}$ tra i cherubini e serafini. La celeberrima poesia che segue immediatamente questo passo, Tanto gentile e tanto onesta pare, conferma gli effetti del suo passaggio e del suo saluto: sembra "che sia una cosa venuta / da cielo in terra a miracol mostrare" (vv. 7-8). La sola sua vista fa tremare e ammutolire - "ogne lingua deven tremando muta, / e li occhi no l'ardiscon di guardare" (vv. 3-4) - e al contempo suscita nel cuore quella dolcezza che, come in ogni esperienza mistica, può capire soltanto colui che la prova:

Mostrasi sì piacente a chi la mira, che dà per li occhi una dolcezza al core, che 'ntender no la può chi no la prova.

(Tanto gentile, vv. 9-II)

A livello strutturale esistono molte corrispondenze fra la Vita Nova e i testi agiografici contemporanei. Hempfer, che le enumera, cita tra l'altro la formula dell'apparuit, l'apparizione qualificata come miracolo, l'analogia con Maria, l'anelito degli angeli al ritorno in cielo dei santi e soprattutto l'analogia con gli angeli. Certo, non si tratta della vita di una santa Beatrice ma dei suoi riverberi sul soggetto narrante (cfr. Hempfer 1982: 35-37); ciò nonostante la concentrazione di tutti questi tratti in un unico personaggio pare tanto significativa quanto l'insistere sulla qualità angelica della sua apparizione e del suo agire. Beatrice è caratterizzata dalla dolcezza che emana dalla sua figura e che il suo saluto, la sua vista e persino la sua mera presenza evocano in colui che l'incontra, pur non osando rivolgerle lo sguardo o on reggendone la vista.

I6. Cfr. "angeli [...] semper vident faciem Patris", citato da Colombo nella sua edizione del testo (Colombo 2008: 184). Per quanto riguarda la locuzione latina alla fine del passo, annota: "citazione paolina d'uso frequente nell' explicit dei trattati sacri, a suggellare le fonti e il tono della Vita Nuova" (ibid.).

17. Cfr. Gorni 1996: 157. Qui anche il rimando a Convivio II v, dove la gerarchia degli angeli viene rappresentata secondo Gregorio Magno, mentre in Pd. XXVIII 98-I35 Dante riprende la versione dello Pseudo-Dionigi. Negli ordini più alti, però, le gerarchie sono identiche. 
Ma Beatrice non è la sola ad essere così strettamente legata all'idea di dolcezza, a quel fenomeno che allude allo stesso tempo e nella stessa misura sia all'esperienza mistica che al suono musicale; anche Amore è un "dolcissimo segnore" (4.3 / ix 3) che appare all'io narrante come una creatura sovrannturale, a volte sotto le spoglie di un pellegrino, altre di un giovane vestito di bianco (cfr. 5.Io / xii 3); all'inizio, nella prima visione, come un essere tanto spaventoso quanto miracoloso, insomma, come un angelo, perché 'ogni angelo - nelle parole di Rilke - è terribile' (trad. Franco Rella), "ein jeder Engel ist schrecklich": "io discernea una figura d'uno segnore di pauroso aspetto a chi la guardasse; e pareami con tanta letizia, quanto a sé, che mirabile cosa era" (I.I4 / iii 3). Come nella letteratura visionaria qualche volta gli angeli parlano una lingua che il visionario non capisce (cfr. Hammerstein 21990: 24), così anche l'io narrante deve constatare che "ne le sue parole dicea molte cose, le quali io non intendea se non poche" (I.I4 / iii 3). Quando l'io capisce il senso delle parole, cioè, quando le può riportare nel suo scritto, Amor utilizza prevalentemente la lingua latina. In quanto lingua liturgica, non solo connota la di lui santità, ma lo collega anche agli angeli che cantano l'Osanna in latino. Non solo nella Vita Nova, ma anche in altri testi poetici, soprattutto delle sacre rappresentazioni, la parte cantata dagli angeli è ancora per lungo tempo in latino, anche se il volgare ${ }^{\mathrm{I} 8}$ si impone sempre di più. Chiedendo pietà, l'io invoca Amore come se fosse un angelo custode: "Amore, aiuta lo tuo fedele" (5.9 / xii 2). In quanto consigliere e messaggero dell'io, esso assume le tradizionali funzioni degli angeli; inoltre fa tremare sia Beatrice che l'io; anche il lessico del testo, l'inusitato "obumbrare", crea un legame con l'angelo dell'Annunciazione del vangelo secondo Luca:

E chi avesse voluto conoscere Amore, fare lo potea mirando lo tremare de li occhi miei. E quando questa gentilissima salute salutava, non che Amore fosse tal mezzo che potesse obumbrare a me la intollerabile beatitudine, ma elli quasi per soverchio di dolcezza divenia tale, che lo mio corpo, lo quale era tutto allora sotto lo suo reggimento, molte volte si movea come cosa grave inanimata $(5.5-6 / \mathrm{xi} 2-3) .{ }^{19}$

18. Secondo Hammerstein, l'angelo si trova alla soglia della sacra rappresentazione e quindi del teatro europeo; segna il luogo di transizione tra il liturgico-corale e il dialogico-drammatico, tra il liturgico-simbolico e il drammatico-realistico. Anche in seguito nel teatro medievale l'angelo che canta rimane una costante liturgica, cosicché il primato del mondo soprannaturale rispetto a quello storico viene rappresentato al tempo stesso scenicamente. Nell'ulteriore evoluzione della sacra rappresentazione, gli angeli esercitano la funzione di quelli che sorvegliano l'ordine dell'azione, che introducono, spiegano e preparano il cammino, sicché anche in questo senso i paralleli con il personaggio di Amore nella Vita Nova risultano evidenti (cfr. Hammerstein 21990: 72-79).

19. Cfr. la nota di Gorni a questo passo: "obumbrare è lessico biblico, o meno persuasivamente classico (Casini); ed è proprio anche nella salutazione angelica: 'Spiritus sanctus superveniet in te, et virtus Altissimi obumbrabit tibi' (Luca I, 35)” (Gorni 1996: 53). 
Amore, come Beatrice, trascina l'io in uno stato di rapimento estatico; non c'è separazione nel loro agire. È ancora più evidente nel momento in cui l'io invoca la donna affinché, in quanto angelo custode, lo sostenga nella "battaglia d'Amore" che si sta svolgendo nel suo cuore, ma dimentica che, come Amore scaccia tutti gli spiriti della vita dal suo corpo, così anche la vista della donna non può essere una difesa in questa battaglia, perché anche lei "disconfiggea la mia poca vita" (5.9/ xvi 5). Vale dunque per tutti e due la descrizione rilkiana dell'agire di tali angeli: "Ich verginge vor seinem / stärkeren Dasein", 'resterei vinto per la sua / [più] forte presenza' (trad. Franco Rella). Sia la donna che Amore procurano "dolcezza" all'io; sia la donna che Amore hanno, com'è noto, la capacità di "nobilitare" o "ingentilire", perché non solo colui che guarda Beatrice diventa, secondo la prima grande canzone, "nobil cosa" (Donne ch'avete intelletto d'amore, v. 36); anche Amore e 'l cor gentil sono una cosa. Questo lo sanno tutti i poeti, e non solo i poeti, a partire dalla lezione di Guinizelli: nel regno di Amore ci sarà inevitabilmente "gentilezza" o "nobiltà". E allo stesso modo, sempre secondo la prima canzone, agisce anche la poesia ispirata o da Amore o da Beatrice, oppure dalla "dolcezza" che sia Amore che Beatrice fanno nascere nell'io:

Io dico che pensando il suo valore,

Amor sì dolce mi si fa sentire,

che s'io allora non perdessi ardire,

farei parlando innamorar la gente.

(Donne ch'avete, vv. 5-9)

Anche le parole dell'io poetante, le sue canzoni, come Beatrice fanno nascere l'amore, e se Amore e "cor gentil" sono "una cosa" sola, fanno nascere al tempo stesso la "gentilezza". Quando le poesie risuonano nella loro "soave armonia", l'amore è presente (cfr. 5.I5 / xii 8); la loro suavitas è segno della presenza d'Amore. ${ }^{20}$ Come Beatrice i canti sono 'cortesi' e 'gentili' (cfr. Ballata, i' voi che tu, vv. 5 e 43); e come Beatrice "non parea figliuola d'uomo mortale, ma di deo" (I.9 / ii 8) ed è dunque figlia d'Amore, così l'io poetante definisce la canzone "figliuola d'Amor" (Donne ch'avete, v. 60). La canzone dice che

20. Secondo Russo, questo passo segnalerebbe addirittura l'identità di "accompagnamento musicale" e "accompagnamento di Amore": "Nel significato sottile di questo passo e nella delicata interpretazione che se ne può dare sta il fulcro della complessa operazione letteraria condotta da Dante: quel che viene qui enunciato è un rapporto di identificazione tra la 'soave armonia', che deve adornare le 'parole per rima', e la presenza di Amore stesso; l'accompagnamento musicale è accompagnamento di Amore; l'elemento melodico perduto nella diversa destinazione della ballata è così come recuperato, reintegrato nella presenza di Amore, personificato quale personaggio, dramatis persona, attante, in un contesto ora finalizzato alla narrazione e alla lettura; l'artificio retorico della prosopopea [...] investe non solo la personificazione dei versi del componimento, ma anche la suavitas della sua melodia, eletta a segno della presenza di Amore o addirittura a suo vivo rappresentante ('ne la quale io sarò tutte le volte che farà mestiere')" (Russo 1985: 253s.). 
Beatrice è così "adorna e pura" che persino Amore si meraviglia di come un essere mortale possa riunire in sé tali attributi. ${ }^{21} \mathrm{D}$ 'altro canto la canzone è a sua volta "adornata" dalla lode di Beatrice che la canta e per tanto è anch'essa caratterizzata da una bellezza angelica. La canzone opera dunque come Beatrice e Amore: avvicina l'amore alla gente; la ammansisce così come il canto di Davide aveva placato l'ira (cfr. Ballata, i' voi che tu, v. I2), e si distingue per "cortesia" e "gentilezza", per "dolcezza" e "beatitudine". In altre parole, la canzone è un angelo.

\section{Cantare come cantano gli ANGeli}

Il tratto più ovvio del carattere angelico dei canti è naturalmente la loro funzione di messaggero, possibile grazie al loro "dolze sono". Tuttavia non è solo nella Vita Nova che nel congedo il poeta-amante incarica la sua canzone di recarsi dalla donna amata per portarle il suo messaggio, cosa che avviene qui nella ripresa della ballata:

Ballata, i' voi che tu ritrovi Amore, e con lui vade a madonna davante, sì che la scusa mia, la qual tu cante, ragioni poi con lei lo mio segnore.

(Ballata, i'voi che tu, vv. I-4)

Ogni strofa della poesia comincia con un riferimento a questo ruolo di messaggero. Nella prima: "Tu vai, ballata, sì cortesemente" e "retrova l'Amor pria". Nella seconda: "Con dolze sono, quando se' con lui, / comincia este parole". Nella terza: "Dille". E infine nella quarta: "E dì a colui ch'è d'ogni pietà chiave", giusto prima che i versi finali sottolineino in un ultimo appello l'importanza della missione:

Gentil ballata mia, quando ti piace, movi in quel punto che tu n'aggie onore.

(Ballata, i' voi che tu, vv. 43-44)

Nella ballata la funzione di messaggero è presente dall'inizio alla fine; e anche nella prima e nella terza canzone ${ }^{22}$ è tematizzata nel congedo: "io son mandata I a quella di cui laude so' adornata” (Donne ch'avete, vv. 62-63), dice la prima alle donne cui è stata inviata, e all'ultima viene affidato il seguente incarico:

2I. Per lo meno per quel che riguarda il senso della parola "pura", anche Gorni ammette qui il carattere angelico: "pura: con armoniche che evocano la natura delle Intelligenze celesti $(P d$. xxix 22-24), o lo stato d'innocenza prima del peccato originale (Adamo nell'Eden 'con vita pura', $P d$. xxvi I40): una Beatrice angelo, oppure 'sine labe originali concepta'” (Gorni I996: I00).

22. Si specchiano dunque in quell'asse centrale dell'intero testo che è la seconda canzone, che procede in un altro modo e che sarà analizzata nella terza parte. Per quanto riguarda il mutuo rispecchiamento delle due canzoni cfr. Martinez i998: I4. 
"Pietosa mia canzone, or va piangendo; / e ritruova le donne e le donzelle" ( $L i$ occhi dolenti, vv. 7I-72).

Ma le poesie non sono soltanto messaggeri come gli angeli, sono soprattutto dolci come il canto angelico; lo indica il "dolze sono", che allude all'accompagnamento musicale, e lo indica il loro straordinario effetto beatificante. In questo 'poetare nuovo', in questo poetare nel 'dolce stil nuovo' la beatitudine non viene dal di fuori, nasce dal poetare stesso. Lo dice l'io nella sorprendente risposta che dà alle donne, accorse, incuriosite e incredule, intorno a lui per sapere "ove sta questa tua beatitudine": "In quelle parole che lodano la donna mia" (Io.8 / xviii 6). Tuttavia anche l'angelo del poetare, come tutti gli angeli, è allo stesso tempo bello e terribile, è desiderato e al contempo infonde paura: "non ardia di cominciare; e così dimorai alquanti dì con disiderio di dire e con paura di cominciare" (Io.II / xviii 9). Però finalmente il nodo nella lingua si scioglie, perché "Amor [...] ditta dentro" ${ }^{23}$ (secondo il concetto che verrà espresso nel Purgatorio), e l'incipit della poesia fluirà quasi da solo, di modo che ancora una volta, come nel momento in cui si contempla Amore, al "pauroso aspetto" s'accompagna la serenità:

la mia lingua parlò quasi come per sé stessa mossa, e disse: Donne, ch'avete intelletto d'amore. Queste parole io ripuosi ne la mente con grande letizia, pensando di prenderle per mio cominciamento; onde poi, [...] pensando alquanti die, cominciai una canzone con questo cominciamento, ordinata nel modo che si vedrà di sotto (IO.I3-I4 / xix 2-3).

La rappresentazione di questo processo poetico corrisponde all'idea esposta nel De vulgari eloquentia: all'iniziale mancanza di ispirazione, e quindi alla necessità di abbeverarsi alla fonte dell'Elicona deve seguire la lunga riflessione grazie alla quale è costruita poi la canzone, devono seguire "opus et labor" o "ar[s] scientiaque". Solo così il poeta può essere elevato metaforicamente al cielo, $\mathrm{o}$ in altre parole la canzone, come un angelo, lo può trasportare al cielo ${ }^{24}$. Al tempo stesso "la lingua [...] quasi da sé stessa mossa" ricorda il cosiddetto dono delle lingue, la glossolalia, in quanto lode e osanna, partecipazione al canto degli angeli. Se nei testi dei mistici non è raro che le apparizioni degli

23. "I' mi son un che, quando, / Amor mi spira, noto, e a quel modo / ch'e' ditta dentro vo significando" ( $P g$. xxvi 52-54). Per quanto riguarda il nodo sciolto cfr. più sotto la citazione delle parole di Bonagiunta da Lucca, che già aveva discusso accaloratamente con Guinizelli sul modo giusto del poetare, ma che adesso riconosce il suo errore.

24. "Caveat ergo quilibet et discernat ea que dicimus, et quando pure hec tria cantare intendit, vel que ad ea directe ac pure secuntur, prius Elicone potatus, tensis fidibus ad supremum, secure plectrum tum movere incipiat. Sed cautionem atque discretionem hanc accipere, sicut decet, hic opus et labor est, quoniam nunquam sine strenuitate ingenii et artis assiduitate scientiarumque habitu fieri potest. Et hii sunt quos Poeta Eneidorum sexto Dei dilectos et $\mathrm{ab}$ ardente virtute sublimatos ad ethera deorumque filios vocat, quanquam figurate loquatur. Et ideo confutetur illorum stultitia qui, arte scientiaque immunes, de solo ingenio confidentes, ad summa summe canenda prorumpunt; et a tanta presumptuositate desistant; et si anseres natura vel desidia sunt, nolint astripetam aquilam imitari" (Dve. II iv 9-II). 
angeli siano ispiratrici di canti (cfr. Hammerstein 21990: 39 sgg. e 5I sgg.), non ci si deve stupire che la canzone, sorta da un moto di quasi auto-ispirazione, sia la prima scritta nel nuovo "stilo de la sua loda" e si presenti subito in veste di inno di lode:

Donne ch'avete intelletto d'amore, i' vo' con voi de la mia donna dire, non perch'io creda sua laude finire, ma ragionar per isfogar la mente.

(Donne ch'avete, vv. I-4)

La canzone non è solo "sua laude", cioè inno di lode, come lo è per definitionem il canto degli angeli, ma di tale canto presenta anche tutte le caratteristiche. L'io vuole cantare questa "laude" insieme con le 'donne che hanno intelletto d'amore', così come i cori degli angeli cantano una voce per segnalare l'unione e l'unanimità della lode (cfr. Hammerstein 21990: 25). La musica stessa delle parole, il gioco di allitterazioni e assonanze di 'i' vo' con voi de la mia donna dire" riproduce il canto una voce. Inoltre, non materialmente ma idealmente, la canzone è sine fine ("non perch'io creda sua laude finire") come infinito è il canto degli angeli, esprimendo così sia il carattere eterno del lodato che quello di coloro che lo lodano, perché questo e quelli non sono sottomessi allo scorrere tempo (cfr. ibid.). ${ }^{25} \mathrm{E}$ come il canto degli angeli è caratterizzato da perfezione,${ }^{26}$ così anche questa prima canzone nello "stilo de la loda" può valere come esempio perfetto del nuovo stile. Ce lo conferma la considerazione del fatto che nel De vulgari eloquentia Dante propone per due volte questa canzone come modello: ${ }^{27}$ una volta del genere lirico, il più alto tra le forme poetiche in volgare (cfr. DVE II viii 7-8), e una secondadell'endecasillabo utilizzato come metro unico, in quanto forma ideale dello stile tragico (cfr. DVE II xii 3). E lo confermerà in misura ancora maggiore Bonagiunta da Lucca, che Dante nel Purgatorio, con fine ironia, eleva a inventore del termine con cui verrà designata la nuova corrente, che d'ora in avanti sarà usato quasi

25. "Sicut sunt immortalia, ita nec eorum laudes aliquo fine clauduntur", scrive Cassiodoro (citato secondo Hammerstein 21990: 25).

26. Cfr. i tratti caratteristici della musica celeste, i quali si trovano ad esempio nelle visioni di Ildegarda di Bingen, la cui 'combinazione affascinante di metafisica luminosa e metafisica sonora' viene paragonata a quella di Dante: "Vollkommenheit, Unsagbarkeit, una voce, sine fine, alter ad alterum, Gemeinsamkeit himmlischer und irdischer Liturgie, Analogie von Engeln und Mönchen" (Hammerstein 21990: 57). Per la concezione teologica dei nove cori degli angeli nel Liber Scivias di Ildegarda di Bingen e per il loro riflesso nel Convivio e nella Commedia cfr. anche Herkommer 2006: 202-206. L'articolo comprende anche numerose illustrazioni di angeli musicanti.

27. Solo la canzone cavalcantiana Donna me prega è menzionata anche lei due volte, ma solo la prima di queste menzioni la porta a modello, insieme a Donne ch'avete, per la canzone costruita idealiter esclusivamente di endecasillabi. La seconda menzione serve piuttosto a fornire un esempio per un trisillabo all'interno di un verso, cosa che appunto succede nella canzone cavalcantiana grazie ad una rima interna (cfr. Dve. II xii 8). 
antonomasticamente, in sostituzione del nome dell'iniziatore della corrente stessa; un riconoscimento postumo da parte di un poeta della vecchia guardia che finalmente comprende tutta la novità e dolcezza della 'canzone angelica':

“[...] Ma di' s'i' veggio qui colui che fore

trasse le nove rime, cominciando

'Donne ch'avete intelletto d'amore'”. [...].

"O frate, issa vegg' io" diss'elli "il nodo

che 'l Notaro e Guittone e me ritenne

di qua dal dolce stil novo ch'i' odo! [...]".

(Pg. XXIV 49-57)

Prima il saluto "dolcissimo" di un'angelica Beatrice procura "beatitudine", ora ci appare la "beatitudine" dell'io nel "dolce stil". La scoperta dell' amor nuovo', della caritas mistica in quanto amore disinteressato, corrisponde alla scoperta di un nuovo stile, dell'autosufficienza della lingua poetica, che non trova più la sua legittimazione pragmatica nel cerimoniale di corte o in altri rituali, evocati all'inizio tramite la menzione del famoso "guiderdone" (cfr. 3.2 / viii 2) ${ }^{28} \mathrm{Al}$ contrario, scegliere l'amore mistico come modello del profano ha dei riverberi anche sul dire di questo amore, sulla concezione della lingua poetica che in qualche modo acquista in santità, come evidenzia Maria Corti in Percorsi dell'invenzione: "se l'estasi amorosa può assumere a modello quella mistica, ciò guida all'ardita estensione nell'ambito dell'esperienza poetica amorosa delle proprietà e quindi del linguaggio dell'ineffabilità, come incapacità di ridicere l'evento amoroso stesso" (Corti 2003: 2I8 sgg.). L'effetto di tale interdiscorsività tra poesia profana e poesia mistica, cioè l'inseparabilità tra la visione mistica e quella lirica nel processo dello scrivere, secondo Corti è doppio, quasi un movimento verso tutte e due le direzioni, quella del 'potenziamento' e quella della 'socializzazione' dell'autore:

da un lato [il modello mistico] potenzia intuizioni dantesche, oggettiva sue esperienze di poeta che si trasfigurano entrando nell'universo della contemplazione. D'altro lato il modello serve a produrre una nuova informazione nel campo della poesia: cioè l'intuizione lirica dell'artista si socializza realizzandosi entro modelli culturali già esistenti e validi, i mistici per l'appunto.

28. Cfr. il commento di Colombo (2008: 95 sgg.) a questa scoperta della "beatitudine" nuova e unica: "È la scoperta, d'ora innanzi stabilmente acquisita, dell'autosufficienza del linguaggio poetico e insieme e per questo della sua natura trascendente, già implicita nella svolta cruciale del cap. $\mathrm{x}[\ldots]$ e generatasi dall'approdo a un nuovo e disinteressato sentimento d'amore. Modello di questo amore è ora la caritas cristiana, segnatamente mistica, le cui proprietà e il cui linguaggio vengono per analogia trasferiti al nuovo sentimento per la beatrice: come quell'amore anche questo è assolutamente gratuito e disinteressato; non ha bisogno, perciò, di alcuna ricompensa; è, in altri termini, pura contemplazione che, in quanto tale, più che raggiunta, è concessa ab alto; come quell'amore anche questo è Verbo, è lingua [...]; di quell'amore questo, infine, riprende le parole che lo dicono, le immagini che lo suggeriscono, i lamenti che ne dichiarano l'inattingibilità per l'assoluta trascendenza." 
La solitaria avventura intellettuale dell'artista viene a collegarsi alle altre del suo tempo (Corti 2003: 220).

Se da un lato Corti, partendo da questa base, qualifica la Vita Nova come il testo che, al pari del De vulgari eloquentia, spreme "come succo una nuova poetica", vale a dire esprime "il senso dantesco del fare scrittura in versi e in prosa" (Corti 2003: 22I), dall'altro però, rispetto al tema 'Dante a la musica' o 'Dante e la canzone' non si discosta da tutti gli studi relativi e soprattutto non approfondisce il contributo particolare della Vita Nova al tema: "lo nome d'Amore è si dolce a udire, che impossibile mi pare che la sua propria operazione sia ne le più cose altro che dolce, con ciò sia cosa che li nomi seguitino le nominate cose, sì come è scritto: 'Nomina sunt consequentia rerum"' (6.4 / xiii 4). Il principio del rapporto necessario tra res e signa del linguaggio poetico, evocato fin dall'inizio nell'allusione al significato del nome di Beatrice ${ }^{29} \mathrm{e}$ qui formulato teoricamente, sarà applicato là dove l'io racconta il suo incontro visionario con Giovanna-Primavera e Beatrice-Amore. Punto culminante sono le parole di Amore che dice: "E chi volesse sottilmente considerare, quella Beatrice chiamerebbe Amore, per molta simiglianza che ha meco" (cfr. I5.3-5 / xxiv 3-5; Corti 2003: 252).

Se però, secondo la definizione della poesia che, come la precedente citazione, ricorre in quasi tutti gli studi menzionati, non escluso il presente, la lirica "nichil aliud est quam fictio rethorica musicaque poita" ( $D V E$ II iv 2);30 se inoltre si può supporre che, benché il trattato sia stato scritto più tardi, questa convinzione fosse valida già all'epoca della Vita Nova, dal momento che ne fa dei chiari riferimenti, allora si dovrebbe poter rintracciare una musicalità della poesia non solo là dove si parla in modo esplicito della relazione tra il nome e la cosa da esso designata, ma anche nell'opera stessa che è una storia d'amore in forma di prosimetrum, in altre parole, una "fictio rethorica musicaque poita" - cioè nell'intera Vita Nova. Tanto più se nel De vulgari eloquentia si legge che il concetto di 'canzone', nell'accezione più ampia che include anche la ballata e il sonetto, può ben significare una poesia accompagnata da musica; ciò nonostante il significato della parola non è l'unico possibile, in quanto 'canzone' può anche designare testi senza accompagnamento musicale. ${ }^{3 \mathrm{I}}$ Inoltre viene affrontata la questione se il termine 'canzone' significhi la

29. "a li miei occhi apparve prima la gloriosa donna de la mia mente, la quale fu chiamata da molti Beatrice li quali non sapeano che si chiamare" (I.2 / ii I).

30. Un'ampia discussione della celeberrima definizione si trova in Paparelli 1960: I-83. In primo luogo però Paparelli si dedica alla definizione della nozione di fictio, i cui significati possibili cerca di chiarire servendosi di etimologia e intertestualità. Solo dopo si concentra anche sugli altri elementi della definizione, innanzitutto su poita. Il ruolo assunto da musica e retorica nella definizione dantesca della poesia non viene menzionato che marginalmente.

3I. "cantio dupliciter accipi potest: [...] sive cum soni modulatione proferatur, sive non" (Dve. II viii 4 ). 
"fabricatio verborum armonizatorum" o la musica stessa. La seconda alternativa viene rifiutata, perché la musica stessa non viene mai definita cantio, ma "sonus", "thonus", "nota" o "melos", ${ }^{2}$ e perché nessun musicista che suoni uno strumento definirebbe la musica da lui prodotta una canzone, eccetto quando non accompagni una poesia. Al contrario, chi mette in musica un testo chiama canzone il risultato del suo lavoro persino quando non viene eseguito ed è conosciuto solo sulla carta. Canzone in senso lato sono dunque tutte le "verba $[\ldots]$ armonizata", che possono essere accompagnate da una melodia (cfr. DVE II viii 5-6). Da questa affermazione consegue che, se da un lato il legame tra la canzone o il poetare e la beatitudine esiste solo a partire dalla scoperta dello "stilo de la sua loda", dall'altro la questione della musicalità della lingua, della sua dolcezza investe la poesia in generale e dunque la Vita Nova nella sua totalità.

Sin dalla prima poesia del prosimetrum è evidente che tale interpretazione è corretta. Nel primo sonetto, indirizzato ai "fedeli d'Amore" e composto in seguito alla visione che rende l'io "come inebriato" dalla dolcezza e dall'estasi, poetare significa superare la mancanza di parole, significa 'trovare' una lingua capace di dire l'ineffabile, perché malgrado lo sbigottimento l'io sente il desiderio di raccontare la sua esperienza, "di farlo sentire a molti li quali erano famosi trovatori in quello tempo" (I.20 / iii 9). Fin da subito dunque dolcezza, estasi e ineffabile da una parte, necessità del dire, dall'altra, si trovano insieme in una tensione creativa, tanto più che l'esperienza interiore (l'essere toccato dall'amore) provoca uno sconvolgimento pari per intensità all'urgenza del dire, come viene più avanti confermato da alcuni versi della canzone cominciata e interrotta:
allor sente la frale anima mia
tanta dolcezza, che 'l viso ne smore,
poi prende Amore in me tanta vertute,
che fa li miei spiriti gir parlando,
ed escon for chiamando
la donna mia $[\ldots]$.
(Si lungiamente, vv. 7-I2)

Secondo le spiegazioni date da Dante nella prima parte del De vulgari eloquentia, gli angeli probabilmente non hanno bisogno della lingua per poter cantare, perché hanno una "promptissimam atque ineffabilem sufficientiam intellectus" grazie alla quale esprimere le loro "gloriosas [...] conceptiones". ${ }^{33}$ Non possedere una lingua non è dunque una mancanza, mentre invece la

32. Cfr. la differenza tra "l'elemento musicale o so" e "quello propriamente testuale o mot" ad es. nella canso dei trovatori (Cerullo 2013: 156).

33. "angeli ad pandendas gloriosas eorum conceptiones habeant promptissimam atque ineffabilem sufficientiam intellectus" (Dve. I ii 3). 
specie umana non ne può fare a meno. ${ }^{34}$ Malgrado la lingua sia uno strumento indispensabile alla comunicazione, anche gli uomini, non solo gli angeli, conoscono l'"ineffabile" (Dante lo dimostra nel Convivio servendosi della canzone Amor che ne la mente mi ragiona), o meglio conoscono "due ineffabilitadi” ( $C v$. III iv I), giacché l'impossibilità di esprimersi è dovuta all'argomento da trattare, quando questo è superiore alle facoltà dell'io, o all'incapacità del soggetto di esprimere adeguatamente quello che sente e prova:

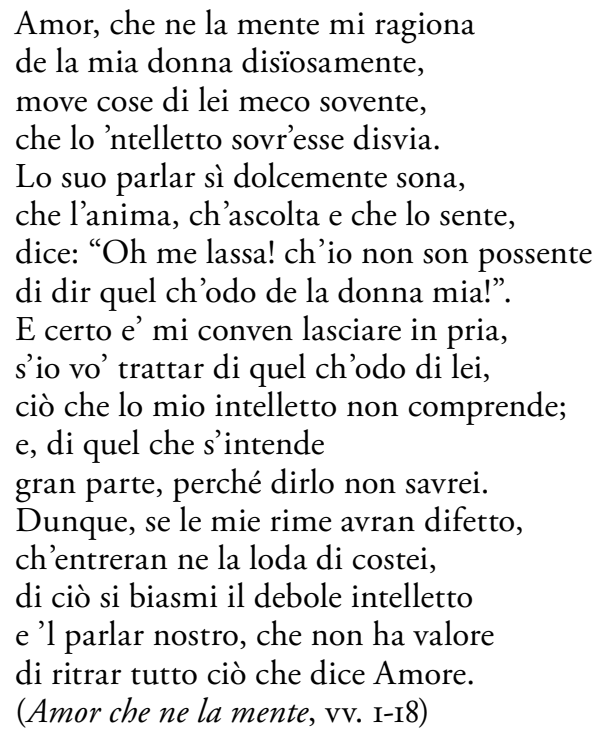

Come la comunicazione degli angeli, anche quella di Amore avviene senza parole. Occorrono allora altri mezzi per poter rendere in lingua umana la dolcezza del suo "parlar"; mezzi che si distinguono per "letizia", "beatitudine" e "infinita dolcezza" - tutti e tre sinonimi della musica nel Paradiso dantesco (cfr. Dovara in Varoli Piazza 1997: 331, nonché Armour 1997: 33-40) e nella Vita Nova caratteristiche di Amore, Beatrice e gli altri angeli. In un primo momento l'io rivolge le sue poesie esclusivamente a "chi lo intende", i "fedeli d'Amore", che sono gli iniziati destinatari del primo sonetto. Ma nemmeno loro riescono a decifrare in modo corretto "lo verace giudicio" dell'enigmatica visione. Anche più tardi, quando l'io allarga la cerchia dei suoi interlocutori, proponendosi di cantare la lode di Beatrice davanti a tutti, anche a coloro che non hanno la possibilità di avere diretta esperienza di lei, la lingua umana si rivela insufficiente ad esprimere l'azione angelica della donna, cosicché la tensione fra quello che deve essere detto e l'indicibile rimane costante:

34. "soli homini datum est loqui, cum solum sibi necessarium fuerit. Non angelis, non inferioribus animalibus necessarium fuit loqui" (Dve. I ii I-2). 
Io dico ch'ella si mostrava sì gentile e sì piena di tutti li piaceri, che quelli che la miravano comprendeano in loro una dolcezza onesta e soave, tanto che ridicere non lo sapeano; né alcuno era lo quale potesse mirare lei, che nel principio nol convenisse sospirare [...; ] propuosi di dicere parole, ne le quali io dessi ad intendere de le sue mirabili ed eccellenti operazioni; acciò che non pur coloro che la poteano sensibilemente vedere, ma li altri sappiano di lei quello che le parole ne possono fare intendere. (I7-3-4 / xxvi 3-4)

\section{LA MUSiCA DElle PAROLE}

Laddove agli altri non resta che il muto sospiro per esprimere la "dolcezza", l'io poetante dispone di altri mezzi. Il sonetto sopra menzionato, Tanto gentile e tanto onesta pare, nella sua "perfetta fusione di logos e melos" 35 ne è tangibile manifestazione, nel momento in cui, secondo il modello delle coblas capfinidas, sfuma, per mezzo dell'allitterazione e della figura etimologica, la linea di demarcazione tra quartine e terzine, pur mantenuta sintatticamente, rendendo così possibile lo scorrere ininterrotto del suono:

e par che sia una cosa venuta

da cielo in terra a miracol mostrare.

Mostrasi sì piacente a chi la mira, che dà per li occhi una dolcezza al core, che 'ntender no la può chi no la prova.

(Tanto gentile, vv. 7-II)

Non è dunque un silenzio senza parole, che udranno i lettori, ma un canto di lode che la poesia rende percepibile grazie alla sua musicalità; così la "dolcezza", tale come è stata sperimentata dall'io per mezzo del "dolze sono", è resa comprensibile a tutti.

Nell'ambito dei canti di lode, l'io formula in modo esplicito il desiderio di trasformare le poesie in angeli per il loro ininterrotto suonare, in messaggeri che portano il messaggio di Beatrice anche tra coloro che non la conoscono. La funzione di messaggeri vale però per il testo intero, essendo questo plasma-

35. "A illustrare questa perfetta fusione di logos e di melos basterebbe citare il sonetto Tanto gentile e tanto onesta pare, forse il più compiuto della Vita nuova. Esso colpisce per la chiarezza della struttura sintattica e per un fluire pacato del periodo che abbraccia, nelle quartine, sempre un'intera strofa e, nelle terzine, l'intero spazio testuale della sirma. Il magico influsso che emana dalla donna "quando altrui saluta" sembra trovar riscontro, sul piano espressivo, nei concatenamenti consecutivi ("tanto ... che", "sì piacente ... che") e ipotetici ("e par ... che", "par ... che") che connotano il discorso emotivamente, in quanto ne sostengono la struttura ritmica e melodica. Beatrice stessa, nel suo incedere pacato e pieno di grazia, provoca negli animi degli astanti un duplice effetto di 'salute' e di stupore, sia conoscitivo, dunque, sia emotivo. In tal modo la donna gentile, salutifera e 'dolce', diventa figura della nuova poetica dantesca" (Güntert I995: I22). Per quanto riguarda la "musicalità segreta" del sonetto Oltre la spera, basata interamente sul suo vocalismo ricco di tensioni, cfr. ibid.: I2I-I22. Güntert rimanda all'"analisi fonematica" dei due sonetti in Sassi 1993. 
to sin dall'inizio dalla dimensione della memoria. Già nel proemio è evocato il "libro de la mia memoria", dal quale il poeta si propone di trarre alcuni dei contenuti da trattare nel "libello", cioè nella Vita Nova. Ma è soprattutto la prima apparizione della donna nell'universo del testo che sottolinea questa dimensione. Ella vi entra in quanto "gloriosa donna de la mia mente", e cioè in quanto donna ricordata, ${ }^{36}$ cosicché solo la "dolcezza" delle poesie, la musica della lingua può far sentire la "dolcezza" emanante dalla sua persona. Grazie a questa componente sonora, alla musicalità, la lingua acquista durata o stabilità, come osserva Dante nel Convivio a proposito del volgare: "Ciascuna cosa studia naturalmente a la sua conservazione". Ma per garantire questa conservazione occorre appunto stabilità, "e più stabilitade non potrebbe avere che in legar sé con numero e con rime" ( $C v \mathrm{I}$, xiii 6$)$, cioè nella dimensione metrica e sonora dei testi.

Un ottimo esempio di questa coesione creata dal suono e della possibilità di esprimere l'ineffabile mediante la "fictio rethorica musicaque poita" è il sonetto Ciò che m'incontra, che segue immediatamente al viavai ininterrotto dei pensieri dialoganti all'interno dell'io. "Mosso da cotali pensamenti" (8.3 / $\mathrm{xv}$ 3), l'io non trova riposo finché non riesce a dare una forma poetica ai pensieri inquieti e dunque inquietanti:

Ciò che m'incontra, ne la mente more, quand'i' vegno a veder voi, bella gioia; e quand'io vi son presso, i' sento Amore che dice: "Fuggi, se l' perir t'è noia".

Lo viso mostra lo color del core, che, tramortendo, ovunque pò s'appoia;

e per la ebrietà del gran tremore le pietre par che gridin: Moia, moia.

Peccato face chi allora mi vide, se l'alma sbigottita non conforta, sol dimostrando che di me li doglia, per la pietà, che l' vostro gabbo ancide, la qual si cria ne la vista morta de li occhi, c'hanno di lor morte voglia.

(Ciò che m'incontra, vv. I-I4)

Di nuovo le allitterazioni trasformano il testo in musica verbale, nei primi due versi, "Ciò che m'incontra, ne la mente more, / quand'i' vegno a veder voi, bella gioia", ma anche in "color del core" e in "le pietre par", dove la "p" non solo è dura come la pietra, ma continua a farsi sentire, a 'ri-sonare' attraverso "Peccato" fino alla successiva allitterazione "per la pietà", la pietà uccisa dal

36. "la quale fu chiamata da molti Beatrice" (I.2 / ii I), scrive di lei l'io, e così pure: "la quale è oggi meritata nel grande secolo" (I.I2 / iii I); fa dunque parte non del suo presente, ma del suo passato. 
"gabbo". E ancora di più sono il poliptoto e la figura etimologica a creare la stabilità sonora e visiva della poesia, sottolineando l'importanza e l'impossibilità del vedere, cui si allude con "veder", "viso", "vide" e "vista" in ognuna delle quartine e terzine, legate tra di loro da questo nesso. In modo analogo "more" e "Moia, moia", tutti e due alla fine dei rispettivi versi e quindi in posizione di rima, formano una cornice che apre e chiude l'ottava ${ }^{37}$ con degli echi in "vista morta" e "morte voglia", collegati dal chiasmo delle identiche consonanti iniziali $v-m$ e $m-v$. Così "vista morta" e "morte voglia" alludono al volere paradossale degli occhi, al loro desiderio di vedere la "bella gioia", pur consapevoli che tale vista li ucciderà; così pure la rima "more" : "Amore" evoca il nesso Eros / Thanatos, per quanto "more" del primo verso alluda piuttosto al dimenticare, creando in questo modo un legame con il tema della memoria nonché con la funzione dei versi stessi, destinati a impedire il 'morire nella mente', vale a dire il dimenticare.

Un altro espediente per creare stabilità mediante il suono che perdura si trova in Amore e 'l cor gentil sono una cosa, poesia che enuncia la tesi in questo primo verso, e la conferma nel secondo per mezzo dell'invocazione all'autorità del "saggio", evidente allusione intertestuale alla famosa canzone $A l$ cor gentil di Guinizelli. Se già la paradossale figura etimologica del quarto verso, "com'alma razional sanza ragione", nella sua suggestiva assurdità rende percepibile l'altra, più grave, assurdità dell'idea di una separazione di "Amor" e "cor gentil", il legame indissolubile di "Amor" e "cor" viene sottolineato anzitutto dalla ripetizione dei due termini in ognuna delle due quartine e nella coppia delle terzine; i due concetti costituiscono anche l'anello di congiunzione con il sonetto successivo, nel quale, come prima nella coppia delle terzine, "Amore" e "core" sono parole rimanti nella prima quartina:

\footnotetext{
Ne li occhi porta la mia donna Amore, per che si fa gentil ciò ch'ella mira; ov'ella passa, ogn'om ver lei si gira, e cui saluta fa tremar lo core.

(Ne li occhi porta, vv. I-4)
}

Un tale connubio sonoro tra le poesie nasce anche là dove all'invito alla lamentazione, Piangete, amanti, poi che piange Amore, dopo la prima morte raccontata nel testo, segue la lamentela Morte villana, di pietà nemica, perché l'apostrofe di questa seconda poesia, nella forma di "villana Morte" (v. 5) era già presente nella prima. E ancora più ovvio sembra quell'altro connubio sonoro, rilevato anche da Gorni, che lega la strofa isolata Si lungiamente m'ha tenuto Amore alla canzone Li occhi dolenti per pietà del core: "L'identità che ostenta-

37. Cfr. inoltre il gioco sonoro con "tramortendo" e "tremore", che conferma al livello dei suoni l'asse semantico della poesia e che mediante la paronomasia crea un legame tra il tremore e la morte. 
no in rima (ore) i capoversi di Gli occhi dolenti e di Si lungiamente connette strettamente l'ultimo testo della prima parte con questo che è il primo della seconda" (Gorni 1996: 176). Se Gorni ne trae la conclusione che si potrebbe trattare di una possibile ispirazione per Petrarca, il legame sonoro tra le due parti 'in vita' e 'in morte di madonna Beatrice' potrebbe altresì alludere all'unità profonda di vita e morte nella Vita Nova, all'in-finitezza dell'operare di Beatrice persino dopo la sua morte, un'infinitezza suggerita tra l'altro dal sine fine del canto di "Amore" e "core", che continua anche oltre la morte terrena.

Comunque la musicalità delle poesie non si crea soltanto "con numero e con rime"; anche il lessico contribuisce in misura non secondaria a tali effetti sonori. Lo aveva già messo in luce la lista delle parole raccomandate nel $D e$ vulgari eloquentia per creare la suavitas: "amore, donna, disio, virtute, donare, letitia, salute, securtate, defesa" (Dve. II vii 5). Questo catalogo costituisce quasi un riassunto delle parole-chiave della Vita Nova e suona come una definizione indiretta di quella "dolcezza" che dal testo promana, una "dolcezza" che grazie al suono stesso delle parole fa sentire agli ascoltatori o lettori quella "dolcezza" provata e raccontata dall'io. Ancora una volta, dunque, il testo illustra l'insieme dei due significati della parola, riproponendo il nesso implicito esistente tra la "dolcezza" del nome e quella dell'operare di Amore: nomina sunt consequentia rerum. In altre parole la scelta dei vocabula è tutt'altro che arbitraria nella poesia in quanto "fictio rethorica musicaque poita". Né mero gioco di suoni, né puro esercizio svincolato dal suono delle parole, tale poesia, come dimostra la doppia "dolcezza" d'Amore, deve piuttosto riunire i due aspetti per poter operare in quanto musica nell'accezione più ampia che il concetto di musica ebbe nel Medioevo.

Più che alcune parole singole, più che la considerazione isolata di "rime" e "numero", è ovviamente la canzone a dimostrare questa unione di 'retorica' e 'musica'. Non a caso è considerata la forma più alta nella gerarchia dei generi poetici, perché a causa della lunghezza, della complessità delle parti legate tra di loro e dell'armonia dell'insieme necessita dell'arte più elevata e al contempo permette a quella stessa arte di dispiegarsi. Proprio come il suono armonioso e la musicalità delle parole sono legati al rapimento mistico, così nell'intera poesia "bellezza" e "bontade" sono legate tra di loro. Quantunque appartengano a livelli diversi - "la bontade è ne la sentenza, e la bellezza è ne l'ornamento de le parole" ( $C v$. II xi 4 ) -, tutte e due, mediante il diletto che possono provocare, offrono un accesso alla poesia. Ė proprio il carattere complesso della "sentenza" che richiede, continua Dante nel Convivio, un'attenzione particolare alla "bellezza", essendo quest'ultima per molti l'unico cammino che porta alla poesia. Si legga il congedo della canzone Voi che 'ntendendo: 
Canzone, io credo che saranno radi color che tua ragione intendan bene, tanto la parli faticosa e forte [...], allor ti priego che ti riconforte, dicendo lor, diletta mia novella: "Ponete mente almen com'io son bella!" (Voi che 'ntendendo, vv. 53-6I)

Queste parole sono rese ancora più esplicite dalla parafrasi nel commento del Convivio:

o uomini, che vedere non potete la sentenza di questa canzone, non la rifiutate però; ma ponete mente la sua bellezza, ch'è grande sì per construzione, la quale si pertiene a li gramatici, sì per l'ordine del sermone, che si pertiene a li rettorici, sì per lo numero de le sue parti, che si pertiene a li musici ( $C v$. II xi 9).

Il carattere particolare della poesia non sta dunque nella rinuncia alla "bontade", ma nel suo stesso valore, nel peso specifico accordato alla "bellezza", alla fusione dell'"eleganza e capacità trascinatrice della retorica più elevata" con la "composizione organica dello strato dei suoni, concepiti come parte essenziale del messaggio poetico" (Pazzaglia 1988: 258).

Un ultimo breve sguardo rivolto alla canzone centrale Donna pietosa e di novella etate può dare un'idea del modo in cui nella complessa opera d'arte che è la canzone "construzione", "ordine" e "numero" si leghino insieme. Essa assume un rilievo speciale sia perché costituisce il centro delle tre canzoni grandi, il centro delle trentuno poesie raccolte nella Vita Nova, sia perché è posta fra il canto di lode Donne ch'avete intelletto d'amore e il canto di lamento Li occhi dolenti per pietà del core; inoltre è caratterizzata da una struttura narrativa all'interno della quale è raccontata ancora una volta la storia della malattia dell'io, che comprende anche la visione di Beatrice già morta. A causa di questo carattere narrativo, la canzone potrebbe sembrare, almeno a prima vista, poco 'lirica' o addirittura 'non-lirica' ${ }^{38} \mathrm{~A}$ un secondo sguardo però, o meglio, a un ascolto più attento del testo, la poesia, che si serve di "construzione", "ordine" e "numero", sembra particolarmente atta a spiegare, a rendere immediatamente percepibile la differenza tra poesia e prosa. Ecco alcuni accenni per fare qualche esempio.

Contrariamente a Voi che 'ntendendo, qui la "sentenza" o "bontade" pare tanto ovvia quanto conosciuta, dal momento che la narrazione dettagliata dei 'fatti' precede la poesia. In questo modo sembra che il poeta faccia soltanto indossare al racconto una veste elegante con lo scopo di raggiungere l'"ornamento de le parole" necessario, la "bellezza" che gli mancava. Un at-

38. Cfr. questi due concetti nel titolo dell'articolo di Cerullo nonché le osservazioni dell'autrice rispetto alla categoria di "lirica" prima del romanticismo (Cerullo 2009: 155). 
tento ascolto del suono delle parole però fa capire come proprio la "musica verbale" nella sua "bellezza" contribuisca alla creazione di una "sentenza" ben più complessa. Già l'incipit della canzone, Donna pietosa e di novella etate, è molto raffinato: con nelle orecchie l'eco della prima canzone indirizzata alle Donne ch'avete intelletto d'amore e grazie all'ellissi dell'articolo davanti a "Donna", leggendo o sentendo la poesia per la prima volta, si ha l'impressione che anche qui si tratti di un'apostrofe e cioè del segnale lirico per eccellenza. ${ }^{39}$ Quest'anfibologia serve a mascherare il carattere narrativo della prima frase, assai complicata e ricca di informazioni:

Donna pietosa e di novella etate, adorna assai di gentilezze umane, ch'era là 'v'io chiamava spesso Morte, veggendo li occhi miei pien di pietate, e ascoltando le parole vane, si mosse con paura a pianger forte.

(Donna pietosa, vv. I-6)

La costruzione molto densa della frase, che riunisce entro la cornice formata dal soggetto "Donna" e il predicato "si mosse [...] a pianger forte" un gran numero di attributi e apposizioni, di frasi relative, gerundi e locuzioni avverbiali, contribuisce a mantenere questa condizione di incertezza, perché crea una tensione molto più alta rispetto alla linearità della frase in prosa. Lì si leggeva:

E dicendo io queste parole con doloroso singulto di pianto, e chiamando la Morte che venisse a me, una donna giovane e gentile, la quale era lungo lo mio letto, credendo che lo mio piangere e le mie parole fossero solamente per lo dolore de la mia infermitade, con grande paura cominciò a piangere (I4.II / xxiii II).

La versione poetica tace sul luogo dell'azione (il letto del malato), rinuncia alla spiegazione introdotta da "credendo che", rendendo più paradigmatica, $\mathrm{ma}$ anche più enigmatica la situazione grazie alla focalizzazione sull'io; d'altro canto espande l'introduzione della "donna giovane e gentile", che invece è brevissima nella prosa, fino a occupare due versi completi, rivelando in questo modo fin dal principio le parole-chiave dell'intera opera. I versi producono soprattutto una sorta di segnali acustici che attirano l'attenzione con il ricorso alle numerose allitterazioni - "pien di pietate", "le parole", "con paura a pianger”. Inoltre per la prima volta nella Vita Nova (cfr. Gorni 1996: 132) la parola "Morte" è collocata in fine verso, in posizione di rima per cui, rispetto alla prosa, la morte acquista un peso particolare.

39. "Now it is certainly beyond question that the figure of address is recurrent in lyric poetry, to the point of constituting the generic definition of, at the very least, the ode (which can, in its turn, be seen as paradigmatic for poetry in general)" (de Man 1985: 6I). 
I versi seguenti non fanno che confermare questo peso, dato che vi si continua a ripetere la parola sotto molteplici variazioni, di nuovo tramite poliptoto e figura etimologica. Così nel verso 34 si legge: "Ben converrà che la mia donna mora"; il verso 42 termina con le parole: "Morra'ti, morra'ti", e troverà una specie di rispecchiamento nel verso 56: "Morta è la donna tua, ch'era sì bella", e, dopo l'eufemismo di Amore: "vieni a veder nostra donna che giace" (v. 64), un altro nel verso 66: "mi condusse a veder madonna morta". Solo dopo le parole pronunciate da Beatrice, "Io sono in pace" (v. 70), parole che non a caso rimano con "imaginar fallace" (v. 65), l'umiltà" della "donna" si trasmette all'io e la morte appare integrata nel pensiero e nel verso, "dolce" $40 \mathrm{e}$ addirittura - è ancora una volta la rima a sottolinearlo - "cosa gentile":

Io divenia nel dolor sì umile,

veggendo in lei tanta umiltà formata,

ch'io dicea: - Morte, assai dolce ti tegno;

tu dei omai esser cosa gentile,

poi che tu se' ne la mia donna stata,

e dei aver pietate e non disdegno.

(Donna pietosa, vv. 7I-76)

La "dolcezza" del canto, operando come Beatrice e Amore, riesce dunque a trasformare persino la morte. Come Beatrice ingentilisce la "villana Morte", così il "dolze sono" trasforma anche la visione della morte in "amorosa cosa da udire" (I4.I6 / xxiii I6).

Affinché tuttavia il componimento poetico nella sua ultima strofa possa esplicitare questo nuovo stato d'animo e soprattutto possa giungere nel penultimo verso al punto culminante dell' exclamatio, "Beato, anima bella, chi te vede!" (v. 83), inesistente alla fine del racconto in prosa, deve scostarsi nettamente dalla struttura temporale del racconto che pur vuole evocare. Seguendo la logica del prorsus, la prosa comincia con la narrazione dei primi segni della malattia, poi della debolezza che ne consegue fino al delirio febbrile, per arrivare all'invocazione della morte da parte del malato. All'udire la quale la donna che lo cura si mette a piangere, facendo accorrere le altre donne che svegliano il malato e gli rivolgono la parola, perché torni in sé e possa raccontare la sua storia. A differenza del testo in prosa, la canzone comincia e finisce all'interno della vicenda narrata. In altri termini si potrebbe dire che, rispetto alla poesia, lo svolgimento dei fatti in prosa segue un ordine corrispondente alla successione delle strofe $3-4-5-6-\mathrm{I}-2-3$. Questo cambia-

40. Anche nella prosa, la morte viene già apostrofata come "Dolcissima", ma è l'insieme di tutti gli elementi sonori a rendere l'ingentilire' della morte davvero impressionante; più importante per la prosa sembra invece il livello semantico, l'opposizione tra villano e gentile, che a sua volta allude al Dolce stil novo. Cfr. ancora una volta l'analisi di Martinez I998: 2I sgg., la cui linea argomentativa è completamente diversa, ma che sottolinea anche la significativa differenza della parola "Morte" usata in rima o integrata nel verso. 
mento dell'"ordine", dettato dalla retorica secondo la definizione del Convivio, ma non senza conseguenze, come si vedrà, per la "musica", crea dunque una struttura circolare che la prosa non conosce. Dopo aver detto della malattia, del delirio e del ritorno alla coscienza, "allora" l'io pone fine al suo narrare per "poi" farne una canzone. ${ }^{4 \mathrm{I}}$ Di conseguenza la composizione poetica segna il punto finale dell'episodio.

La canzone invece comincia con la "Donna pietosa", che insieme alle altre donne rivolge la parola all'io, e termina con il verso: "Voi mi chiamaste allor, vostra merzede" (v. 84). Tale verso è l'unico che segue all'apostrofe dell'"anima bella" e alla beatitudine di colui che la vede. Con questa apostrofe alle donne presenti, si torna all'inizio, alla "donna giovane e gentile, la quale era lungo lo [suo] letto" e alle "altre donne che per la camera erano" (I4.IIs. / xxiii IIs.). In questo senso, la poesia, versus invece di prorsus, fa esattamente il contrario del programma annunciato nella prosa con "cominciandomi dal principio infino a la fine". Il componimento poetico invece di far concludere l'episodio, fa in modo che si possa immediatamente ricominciarlo dall'inizio, ad infinitum oppure sine fine, come il canto degli angeli, come l'Osanna infinito che essi intonano:

Levava gli occhi miei bagnati in pianti, e vedea, che parean pioggia di manna, li angeli che tornavan suso in cielo, e una nuvoletta avean davanti, dopo la qual gridevan tutti: Osanna.

(Donna pietosa, vv. 57-6I)

L'inizio del coro angelico proprio a questo punto della canzone è dovuto alla ristrutturazione della storia, per cui la linearità dei fatti nella prosa è sostituita con una forma circolare, modificando essenzialmente la temporalità della poesia, rispetto a quella della prosa, ma anche, in certo qual modo, sacralizzandola, in quanto solo gli esseri terreni o profani sono sottoposti al tempo e cioè all'inevitabile avvicinamento alla morte. In particolare, la nuova strutturazione permette di sacralizzare il canto mediante alcuni noti effetti gematrici, che la prosa invece ignora. Dunque non è un caso che l'Osanna in esaltazione di Beatrice venga cantato proprio al verso 6I. Già prima, nel corso del serventese perduto o fittizio, il nome di Beatrice era stato menzionato al nono posto tra le 60 più belle donne della città (cfr. 2.II / vi 2), in un chiaro riferimento all'unica, alla più bella e alla più cara del Cantico dei Cantici, 'coperta di lodi' da 60 regine (cfr. Cantico dei Cantici, 6, 8-9). Nel sistema gematrico il 6I è il

4I. "Allora, cominciandomi dal principio infino a la fine, dissi loro quello che veduto avea, tacendo lo nome di questa gentilissima. Onde poi, sanato di questa infermitade, propuosi di dire parole di questo che m'era addivenuto, però che mi parea che fosse amorosa cosa da udire; e però ne dissi questa canzone: Donna pietosa e di novella etate" (I4.I6 / xxiii I6). 
numero che corrisponde al nome di Beatrice, proprio come il 42 risulta dalle lettere che formano il nome di Dante. In questa logica pare altrettanto poco casuale il fatto che il "Morra'ti, morra'ti" si trovi proprio al verso 42 e che la canzone intera sia composta da 84 , cioè due volte 42 , versi. In questo modo i rimandi numerologici svelano che la vecchia vita di questo Dante è finita, ma anche che a questa vita seguirà una "vita nova", annunciata già nel proemio e cantata a partire dalla seconda metà della canzone, dopo la considerazione della "frale vita" ("a veder mio colore", v. 2I), quasi 'nel mezzo del cammin', e dopo il Memento mori del verso 42 in un inarrestabile elevazione che dal lutto delle donne espresso in lamentazioni, attraverso l'ascensione di Beatrice con il coro degli angeli culmina nella sua intronizzazione nell'“alto regno" (v. 82) e nella beatitudine del "Beato, anima bella, chi te vede!" (v. 83) - che altro non è se non una parafrasi del nome della "beatrice".

Ovviamente non è strettamente necessario cogliere nell'orientamento gematrico di questo come di altri testi danteschi un'allusione alla duplice concezione della musica nel Medioevo, quella liturgica e quella caratterizzata dal numerus, concezione spiegata dettagliatamente da Hammerstein. Sembra invece indubbio che questi elementi gematrici siano legati ad una certa concezione dell'ordine del cosmo, e che Dante conoscesse benissimo tanto l'idea dei nove cieli mobili che fanno nascere la musica delle sfere quanto quella dei nove cori degli angeli appartenenti alle tre gerarchie, dato che è lui stesso, sia nel Convivio che nel Paradiso, a stabilire delle corrispondenze tra gli ordini degli angeli e le sfere celesti mobili, corrispondenze di cui parla, secondo Dante, anche il Salmista: "Li cieli narrano la gloria di Dio" (Cv. II v I2-I3). ${ }^{42}$ Corrispondenze simili esistono tra le sfere celesti e le artes liberales, ad esempio tra la musica e il Cielo di Marte, il cui legame ha due ragioni d'essere. ${ }^{43}$

Da un lato, il cielo di mezzo viene associato alla musica per via della sua posizione e delle belle relazioni che ne risultano, il che al tempo stesso po-

42. A. Mellone, "Gli angeli in Dante", in ED, s. v. Angelo, p. 269; cfr. inoltre i compiti dei nove cori degli angeli, legati a certi testi biblici come il Padre nostro, in Knoch 2006: 27.

43. "lo cielo di Marte si può comparare a la Musica per due proprietadi: l'una si è la sua più bella relazione, ché, annumerando li cieli mobili, da qualunque si comincia o da l'infimo o dal sommo, esso cielo di Marte è lo quinto, esso è lo mezzo di tutti, cioè de li primi, de li secondi, de li terzi e de li quarti. L'altra si è che esso [...] Marte dissecca e arde le cose, perché lo suo calore è simile a quello del fuoco; e questo è quello per che esso pare affocato di colore, quando più e quando meno, secondo la spessezza e raritade de li vapori che 'l seguono [...;] l'accendimento di questi vapori significa morte di regi e transmutamento di regni; però che sono effetti della segnoria di Marte [...]. E queste due proprietadi sono ne la Musica, la quale è tutta relativa, sì come si vede ne le parole armonizzate e ne li canti, de' quali tanto più dolce armonia resulta, quanto più la relazione è bella; la quale in essa scienza massimamente è bella, perché massimamente in essa s'intende. Ancora: la Musica trae a sé li spiriti umani, che quasi sono principalmente vapori del cuore, sì che quasi cessano da ogni operazione; sì è l'anima intera, quando l'ode, e la virtù di tutti quasi corre a lo spirito sensibile, che riceve lo suono" ( $C v$. II xiii 20-24). 
trebbe essere un indizio dell'importanza attribuita ad una poesia posta nel mezzo della Vita Nova, e quindi alla posizione di un elemento particolare rispetto al suo insieme, e in generale alle relazioni degli elementi tra di loro. Quello che, dall'altro lato, sembra però soprattutto interessante nel contesto del tema 'Dante e la musica' è l'altro parallelismo scoperto dal poeta tra Marte e la musica. Se la musica, che per Dante include qui esplicitamente "parole armonizzate" e canti, proprio come Marte attira a sé tutti gli "spiriti umani", essa ottiene lo stesso effetto che Beatrice produce già all'inizio della Vita Nova e poi ripetutamente fino alla famosa e decisiva "scena del gabbo": la musica e le sfere provocano tanta "dolcezza" o "soavità" quanta Beatrice e gli (altri) angeli. Proprio questa dolcezza però supera la sfera umana, se è "per soverchio di dolcezza" che il corpo dell'io si può muovere soltanto "come cosa grave inanimata" ogni volta che la sua beatitudine "passava e redundava la [sua] capacitade" (5.7/ xi 4), oppure se dopo l'episodio del "gabbo" dice al suo amico: "Io tenni li piedi in quella parte de la vita di là da la quale non si puote ire più per intendimento di ritornare" (7.8 / xiv 8). Marte e musica attraggono a sé i vapori e così provocano "morte di regi e transmutamenti di regni", proprio come l'io qui, dopo la sua morte simbolica, ${ }^{44}$ entra in un regno - dell'essere come del cantare - diverso.

Come l'uomo, a differenza degli angeli, non è un essere puramente spirituale, ma composto di corpo e spirito, così, differenziandosi anche in questo da loro, non può comunicare immediatamente e senza linguaggio; ha bisogno delle parole che sono composte da "signum rationale et sensuale", come è la sua stessa natura di uomo. ${ }^{45}$ Comunque se il linguaggio poetico - proprio quello dunque che Dante mette in relazione con la musica - non solo come ogni linguaggio si compone dei due aspetti, ma li accorda all'unisono in quelle "parole armonizzate" che sono le liriche in quanto "fictio rethorica musicaque poita", allora la poesia, procurando "beatitudine" o "dolcezza" - come fanno Beatrice, gli angeli e la musica - è angelos lei stessa, non solo nel senso che trasmette un 'messaggio' o la "bontade", ma anche e prima di tutto nel senso che è messaggio lei stessa: "bellezza" in quanto "bontade". Anche se Dante nel passo in questione separa l'una dall'altra, ascrivendo un diletto più grande alla "bontade" del "sermone", ciò nonostante altri passi rivelano in modo più che evidente che la poesia non si contenta di essere "sermone", ma lega i due aspetti del linguaggio così indissolubilmente che in ultima istanza risulta intraducibile, secondo la posizione presa da Dante nel Convivio: "sappia ciascu-

44. Per quanto riguarda questa morte simbolica dell'io e le citazioni bibliche evocate da questo passo cfr. il commento di Gorni 1996: 72.

45. "Oportuit ergo genuas humanum ad comunicandas inter se conceptiones suas aliquod rationale signum et sensuale habere: [...] nam sensuale quid est in quantum sonus est; rationale vero in quantum aliquid significare videtur ad placitum" (Dve. I iii 2-3). 
no che nulla cosa per legame musaico armonizzata si può de la sua loquela in altra transmutare sanza rompere tutta sua dolcezza e armonia" ( $C v$. I vii I 4$){ }^{46}$

In quanto unità indissolubile e armonica dello spirituale e del sensuale però, questa musica verbale che è la poesia si fa immagine di quell'armonia dell'anima, di quell'unità di corpo e mente che è la musica humana secondo la definizione di Boezio, veduta o sentita dall'io della Vita Nova allorquando trova la "beatitudine" nelle "parole che lodano la donna mia" e grazie ad esse riesce paradossalmente a superare la fondamentale ed inevitabile "ineffabilitade". È proprio in questo senso che la poesia è la realizzazione della "dolcezza" - per quanto rimanga sempre il punto finale e trascendente anelato da quello "spirito peregrino" che è l'uomo finché vive. Qualche volta, però, la "dolcezza" della finzione retorico-musicale riesce a portarlo già in vita "oltre la spera", là dove in quanto linguaggio degli angeli può essere sentita anche sulla terra, vale a dire nella poesia.

\section{RIFERIMENTI BIBLIOGRAFICI}

\section{Testi di riferimento}

Barbi, M. (a cura di), 1932, Dante Alighieri, 1932, Vita Nuova, Firenze, Bemporad.

Colombo, M. (a cura di), 2008, Dante Alighieri, Vita Nuova, Milano, Feltrinelli.

Gorni, G. (a cura di), 1996, Dante Alighieri, Vita Nova, Torino, Einaudi.

Inglese, G. (a cura di), 1993, Dante Alighieri, Convivio, Milano, Rizzoli.

Inglese, G. (a cura di), 1998, Dante Alighieri, [De vulgari eloquentia] L'eloquenza in volgare, testo latino a fronte, Milano, Rizzoli.

Mengaldo, P. V. (a cura di), 1968, Dante Alighieri, De vulgari eloquentia, Padova, Antenore.

Simonelli, M. (a cura di), 1966, Dante Alighieri, Convivio, Bologna, Pàtron.

\section{Studi}

Armour, P., 1997, "La musica nel paradiso terrestre dantesco", in Moestrup, Spore e Jørgensen 1997: 33-40.

Barański, Z. G., I997, "Teoria musicale e legittimazione poetica nella Commedia di Dante", Moestrup, Spore e Jørgensen 1997: 75-82.

Basile, G., 1997, "Musicalità e soavità nella 'Divina Commedia”, in Moestrup, Spore e Jørgensen I997: II9-I24.

46. Se dunque, secondo quanto riassume Pazzaglia, anche lo "strato dei suoni" viene concepito come "parte essenziale del messaggio poetico", se quindi, come in questo passo della Vita Nova che si serve dell'esempio di Amore per dimostrare l'accordo tra suono significante e oggetto significato, è evidente la convinzione che il linguaggio abbia la capacità di esprimere la verità anche mediante la sua componente sensuale o sonora, allora se ne può concludere che "questa eufonia o impasto armonico dei suoni del testo poetico, riconducibile alla musica nell'accezione più vasta che la parola ebbe nel Medioevo, appariva a Dante come una prima e necessaria manifestatio dell'idea della 'bontade' che la 'bellezza' celava e, insieme, svelava” (Pazzaglia 1988: 258 sgg.). 
Boyde, P., I984, "Gli angeli", in Id., L'uomo nel cosmo. Filosofia della natura e poesia in Dante, Bologna, Il Mulino [versione inglese I98I], pp. 287-329.

Bruggisser-Lanker, T., 2006, "Engelsmusik und Marienverehrung. Die Engelweihe der Gnadenkapelle zu Maria Einsiedeln”, in Engel Teufel und Dämonene. Einblicke in die Geisterwelt des Mittelalters, a cura di H. Herkommer e R. C. Schwinges, Basel, Schwabe, pp. I77-198.

-, 20Iо, Musik und Tod im Mittelalter. Imaginationsräume der Transzendenz, Göttingen, Vandenhoeck und Ruprecht.

Cappuccio, C., 2005, "Gli effetti psicologici della musica sui personaggi del Purgatorio", Tenzone, 6, pp. 35-80.

—, 2008, "Strutture musicali del cielo del Sole: Dante e Beatrice al centro della danza dei beati", Tenzone, 9, pp. I47-I78.

—, 2009, "Aure musicali in Dante", Cuadernos de Filología Italiana, I6, pp. I09-I36.

Carrai, S., 2006, Dante elegiaco. Una chiave di lettura per la Vita Nova, Firenze, Olschki.

Certeau, M. de, 1984, Le parler angélique. Figures pour une poétique de la langue, Paris, Institut National de la Langue Française.

Cerullo, S., 2009, "Lirica e non-lirica nella poesia dei trovatori: intersezioni generiche e metrico-formali tra salut e canso", in La lirica romanza del Medioevo. Storia, tradizioni, interpretazioni, a cura di F. Brugnolo e F. Gambino, Padova, Unipress, pp. I55-I74.

Colombo, M., 1987, Dai mistici a Dante. Il linguaggio dell'ineffabilità, Firenze, La Nuova Italia.

Corti, M., 2003, Scritti su Cavalcanti e Dante. La felicità mentale, Percorsi d'invenzione e altri saggi, Torino, Einaudi.

De Benedictis, R., 200o, Ordine e struttura musicale nella Divina Commedia, Fucecchio (Firenze), European Press Academic Publishing.

De Man, P., I985, "Lyrical Voice in Contemporary Theory: Riffaterre and Jauss", in Lyric Poetry. Beyond New Criticism, a cura di C. Hosek e P. Parker, Ithaca, Cornell University Press, pp. 55-72.

Dovara in Varoli Piazza, I., I997, "Intendimento dantesco della musica come espressione del sublime", in Moestrup, Spore e Jørgensen I997, vol. I: 33I-340.

Friedrich, H., 1964, Epochen der italienischen Lyrik, Frankfurt, Klostermann.

Ginsberg, W., 1999, Dante's Aesthetics of Being, Ann Arbor, The University of Michigan Press.

Gorni, G., 2006, "La Vita Nova dalla Donna Gentile a Beatrice, con un excursus sulla doppia redazione del libello", Deutsches Dante-Jahrbuch, 8I, pp. 7-26.

Güntert, G., I995, "Le rime della Vita Nova", Letture Classensi, 24, pp. I09-I28.

Hammerstein, R., 21990, Die Musik der Engel. Untersuchungen zur Musikanschauung des Mittelalters, Bern, Francke.

Heisenberg, W., I989, Ordnung der Wirklichkeit, München - Zürich, Piper.

Hempfer, K. W., I982, "Allegorie und Erzählstruktur in Dantes Vita Nuova", Deutsches Dante-Jahrbuch, 57, pp. 7-39.

Herkommer, H., 2006, "Sphärenklang und Höllenlärm, Lächeln oder Fratzen. Zur sinnenhaften Wahrnehmung der Geistwesen", in Engel Teufel und Dämonene. Einblicke in die Geisterwelt des Mittelalters, a cura di H. Herkommer e R. C. Schwinges, Basel, Schwabe, pp. 199-224.

Iannucci, A. A., I989, "Musica e ordine nella Divina Commedia (Purgatorio II)", in Studi Americani su Dante. Introduzione di Dante della Terza, a cura di G. C. Alesso 
e R. Hollander, Milano, Franco Angeli, pp. 87-III.

Jones, N. A., 20Io, "Music and the maternal voice in Purgatorio xix", in L. C. Dunn e N. A. Jones, Embodies voices. Representing Female Vocality in Western Culture, Cambridge, Cambridge University Press [II994], pp. 35-49.

King, M., 1974, "Ut Musica Poiesis: the Effect of Music on Italian Poetics in the Cinquecento", Italian Quarterly, 70, pp. 49-62.

Knoch, W., 2006, "Die Engellehre Bernhards von Clairvaux: Vergessene Einsichten aus der Blüte mittelalterlicher Mönchstheologie”, Das Mittelalter, II, pp. IO-28.

Kropfinger, K., I988, "Dante e l'arte dei trovatori”, in Pestalozzi 1988: I30-I74.

Kuhn, B., 2009, "Beatrice und die anderen Engel: die Musik (in) der Vita Nova", Deutsches Dante-Jahrbuch, 84, pp. 77-IO9.

Martinez, R. L., I998, "Mourning Beatrice: The Rhetoric of Threnody in the Vita nuova", Modern Language Notes, II3, pp. I-29.

Moestrup, J., P. Spore e C.-K. Jørgensen (a cura di), I997, Atti del XIV Congresso dell'Associazione Internazionale per gli Studi di Lingua e Letteratura Italiana (Odense, I-s luglio I99I), 2 vol., Letteratura italiana e musica, Odense, Odense University Press.

Monterosso, R., 1965, "Musica e poesia nel De vulgari eloquentia", in Dante. Atti della giornata internazionale di studio per il VII centenario (Ravenna, 6-7 marzo 1965), a cura della Società di Studi Romagnoli, Faenza, Fratelli Lega, pp. 83-Ioo.

Morelli, G., 1989, "Immagini dell'udire. (A proposito di Dante musicus)", in In cantu et in sermone. For Nino Pirrotta on his 8oth Birthday, a cura di F. Della Seta e F. Piperno, Firenze [et al.], Olschki [et al.], pp. 7-39.

Paparelli, G., I960, “Fictio. La definizione dantesca della poesia”, Filologia Romanza, VII, pp. I-83.

Pasquini, E., I995, "Il dolce stil novo", in Storia della letteratura italiana, vol. I, Dalle origini a Dante, a cura di Enrico Malato, Roma, Salerno, pp. 649-72I.

Pazzaglia, M., 1967, Il verso e l'arte della canzone nel De vulgari eloquentia, Firenze, La Nuova Italia.

—, I986, "L'universo metaforico della musica nella Divina Commedia", Letture Classensi, I5, pp. 79-97.

—, I988, "Musica e metrica nel pensiero di Dante", in Pestalozzi 1988: 257-290.

Pestalozzi, L. (a cura di), I988, La musica nel tempo di Dante. Ravenna, Comune di Ravenna, Opera di Dante, Musica/Realtà (I2-I4 settembre I986), Milano, Edizioni Unicopli.

Petrocchi, G., 1994, "La dottrina degli angeli", in Id., Itinerari danteschi. Premessa e cura di Carlo Ossola, Milano, Franco Angeli, pp. 279-292.

Pirrotta, N., 1968, "Dante Musicus: Gothicism, Scholasticism an Music", Speculum, 43.2, pp. $245-257$.

Pistelli Rinaldi, E., I968, La musicalità di Dante, Firenze, Le Monnier.

Richter, L., I988, "Dante e la musica del suo tempo", in Pestalozzi I988: 55-II2.

Russo, V., I985, "'Dolze sòno' e prosopopea di Amore: 'Ballata, i’ voi' (VN, XII IO-I5), 'sive cum soni modulatione [...] sive non' (DVE, II 84 )", Filologia e critica, IO, pp. 239-254.

—, I988, "Musica/Musicalità nella struttura della Commedia di Dante", in Pestalozzi I988: 33-54.

Sanguineti, E., I988, "Canzone sacra e canzone profana”, in Pestalozzi I988: 206-22I.

Santagata, M., 1999, Amate e amanti. Figure della lirica amorosa fra Dante e Petrarca, Bologna, Il Mulino. 
Sassi, G., 1993, La mente intralinguistica. L'instabilità del segno: anagrammi e parole dentro le parole, Genova, Marietti.

Schneider, F., 20Io, "Ancora su 'Dante Musicus': Musica e dramma nella Commedia", Studi Medievali e Moderni, I4, pp. 5-24.

—, 20I2, "Brevi note su musica e dramma nella Commedia", Modern Language Notes, I27, Supplement, pp. IIO-II8.

Slawinski, M., 2008, "Celestial Discords: The Music of Dante's Paradise", Comparative Critical Studies, 5.I, pp. 57-80.

Stevens, J. E., 1968, "Dante and Music", Italian Studies, 23, pp. I-I8.

Verdicchio, M., 20Io, "Heaven of Mars: Music", in Id., The Poetics of Dante's "Paradiso". Toronto - Buffalo - London, University of Toronto Press, pp. 77-IO7.

Wehle, W., 1986, Dichtung über Dichtung. Dantes Vita Nuova: die Aufhebung des Minnesangs im Epos, München, Fink.

Williamson, B., 2013, "Sensory Experience in Medieval Devotion: Sound and Vision, Invisibility and Silence", Speculum, 88.I, pp. I-43. 
